\title{
Geometry of giant star model atmospheres: a consistency test
}

\author{
U. Heiter and K. Eriksson
}

\author{
Department of Astronomy and Space Physics, Uppsala University, Box 515, 75120 Uppsala, Sweden \\ e-mail: [Ulrike.Heiter;Kjell.Eriksson]@astro.uu.se
}

Received 27 January 2006 / Accepted 9 March 2006

ABSTRACT

\begin{abstract}
Aims. We investigate the effect of a geometric inconsistency in the calculation of synthetic spectra of giant stars. Methods. Spectra computed with model atmospheres calculated in spherical geometry while using the plane-parallel approximation for line formation calculations $\left(s_{-} p\right)$, as well as the fully plane-parallel case $\left(p_{-} p\right)$, are compared to the consistently spherical case $\left(s \_s\right)$.

Results. We present abundance differences for solar metallicity models with $T_{\text {eff }}$ ranging from 4000 to $6500 \mathrm{~K}$ and $\log g$ from 0.5 to 3.0 [cgs]. The effects are smaller for $s_{-} p$ calculations $\left(-0.1\right.$ dex in the worst case) than for the $p_{-} p$ case (up to +0.35 dex for minority species and at most -0.04 dex for majority species), both with respect to the $s_{-} s$ case. In the $s_{-} p$ case the differences increase slightly with temperature, while in the $p_{-} p$ case they show a more complex behaviour. In both cases the effects decrease with increasing $\log g$ and increase with equivalent width.

Conclusions. Within the parameter range of F, G and K giants, consistency seems to be less important than using a spherical model atmosphere. The abundance differences due to sphericity effects presented here can be used for error estimation in abundance studies relying on plane-parallel modelling.
\end{abstract}

Key words. stars: atmospheres - stars: late-type - techniques: spectroscopic - line: formation

\section{Introduction}

Classical abundance analyses are usually based on model atmospheres calculated assuming plane-parallel geometry. This means that radiative transfer is solved in only one depth variable, neglecting the curvature of the atmosphere. For most stars this is justified because the extension of the atmosphere is negligibly small compared to the stellar radius. Extreme cases are cool giants and supergiants, where geometry effects due to the thickness of the atmosphere have to be taken into account. They have been studied accordingly well in the literature.

Sphericity effects on structures, low resolution spectra and colors of cool $\left(T_{\text {eff }} \leq 4000 \mathrm{~K}\right)$ giants and supergiants have been studied for example by Plez et al. (1992). They showed that for one of their hottest models with $\left(T_{\text {eff }}, \log g^{1}\right.$, mass $)=(3800 \mathrm{~K}$, $\left.1.0,1 M_{\odot}\right)$, the changes in temperature structure when releasing the plane-parallel approximation are relatively small (about $35 \mathrm{~K}$ at $\log \tau_{\text {Ross }}=-4$, the atmospheric extension being about $4 \%$ ). Plez (1990) discussed effects of atmospheric extension on the determination of effective temperature, surface gravity and abundances of cool supergiants $\left(T_{\text {eff }} \leq 4500 \mathrm{~K}\right)$. He noted that the effects are not linear with temperature and gravity.

Spherical model atmospheres for a large range of giant star temperatures have been presented by Hauschildt et al. (1999). They also presented changes in spectral flux with respect to plane-parallel models. For a model with $\left(T_{\text {eff }}, \log g\right)=(5600 \mathrm{~K}$, $0.0)$ they show for example that the change in central depth of individual spectral lines can be up to $15 \%$.

$\mathrm{F}$ to $\mathrm{K}$ giants are commonly used to study abundances of stellar systems, mainly due to their intrinsic brightness. With respect to atmosphere geometry they represent borderline cases and are therefore usually analysed using plane-parallel radiative

\footnotetext{
${ }^{1} \log g$ values are given in cgs units throughout the paper.
}

transfer. Many examples for recent large scale abundance analyses of giant stars can be found in the literature. Cepheid stars at various pulsation phases were studied by Luck \& Andrievsky (2004). These stars are used to determine the Galactic abundance gradient (Andrievsky et al. 2004). Another example is the analysis of probable giant star planet hosts (Takeda et al. 2005). Furthermore, red giants are frequently contained in samples of Galactic halo stars, which are used to study Galactic chemical evolution and nucleosynthesis by means of classical abundance analysis. Earlier examples are the large scale high resolution abundance studies of metal-poor stars by McWilliam et al. (1995, 31 stars with $\log g \lesssim 2.5$ ) and Ryan et al. (1996, 7 stars with $\log g \lesssim 2.0$ ), whose results have been used by Travaglio et al. (2001) in their study of the chemical evolution of the Galactic halo. More recently, Simmerer et al. (2004) determined abundances of $\mathrm{Fe}$, La and Eu for stars over a wide range of metallicities. A substantial fraction of those are giant stars in the $\log g$ range of 0.5 to 2.0 , all with $[\mathrm{Fe} / \mathrm{H}] \lesssim-1$ dex.

In recent years, grids of model atmospheres calculated in spherical geometry have become available to an increasing extent (e.g. Gustafsson et al. 2003; Hauschildt et al. 1999). However, line formation will probably continue to be computed in plane-parallel geometry in abundance analysis procedures. In this paper, we investigate the effect of such an inconsistency on synthetic spectra and abundances of giant stars. We then compare to the effects due to using a consistent, but plane-parallel combination of model atmosphere and spectrum synthesis.

Figure 1 shows the atmospheric extension of spherical MARCS models (see Sect. 2) with $\xi_{\mathrm{t}}=2 \mathrm{~km} \mathrm{~s}^{-1}$, solar abundances and various temperatures and gravities as a function of bolometric magnitude. Here, the extension is defined as the difference in radius at the points in the atmosphere where $\tau_{\text {Ross }}=$ $10^{-5}$ and 1 relative to the radius at $\tau_{\text {Ross }}=1$. The bolometric 


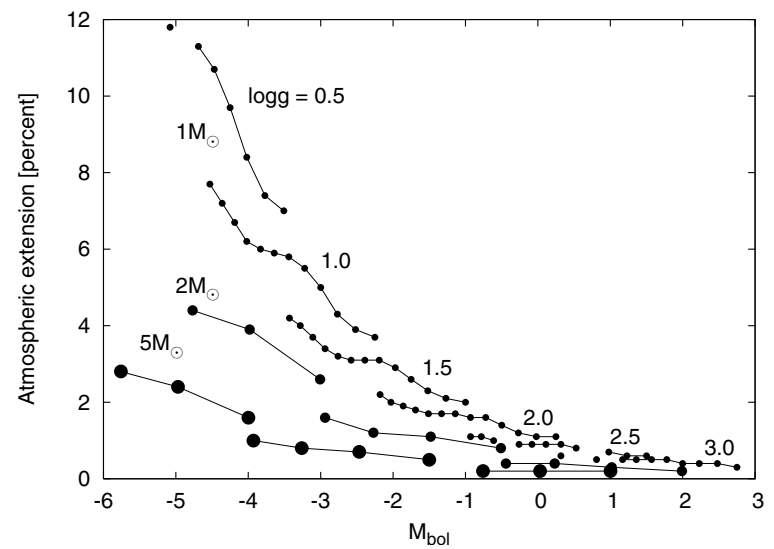

Fig. 1. Atmospheric extension $\left(\frac{r\left(\tau_{\text {Ross }}=10^{-5}\right)}{r\left(\tau_{\text {Ross }}=1\right)}-1\right)$ of spherical models with $\xi_{\mathrm{t}}=2 \mathrm{~km} \mathrm{~s}^{-1}$ and solar abundances as a function of bolometric magnitude for $M=1,2$, and $5 M_{\odot}$ as indicated by increasing symbol size. Models with equal $\log g$ are joined by lines, except where certain $T_{\text {eff }}$ values are missing, and are labelled with their $\log g$ value. Model temperatures range from 4000 to $7000 \mathrm{~K}$ in steps of $250 \mathrm{~K}$ for $M=1 M_{\odot}$ and $1000 \mathrm{~K}$ for $M=2$ and $5 M_{\odot}$, increasing from right to left along lines.

magnitude has been calculated from the model fluxes on the scale defined by IAU Commission 36 (Andersen 1999). This figure can be directly compared to Fig. 1 of Plez et al. (1992). It shows that atmospheric extensions increase relatively rapidly with effective temperature for $\log g<2$. One can also see small local maxima at about $5250 \mathrm{~K}$, similar to the ones at $3200 \mathrm{~K}$ found by Plez et al. (1992). These features in the shape of lines of equal $\log g$ can probably be attributed to a decrease in partial pressure gradient of diatomic molecules such as $\mathrm{CO}$ or $\mathrm{CN}$. The same plot for models with abundances corresponding to halo stars with $[\mathrm{Fe} / \mathrm{H}]=-2$ dex looks very similar, except that the aforementioned maxima are not present. This is in accordance with the low metallicity where molecules are not as important.

Figure 2 shows how the temperature structures of spherical models with solar abundances in the parameter range investigated here differ from plane-parallel ones. The dilution of the radiation field in spherical geometry causes the spherical model to be cooler than the plane-parallel one above an optical depth of $\approx 1$, and the temperature difference increases outwards.

The differences seen for the hotter models at large optical depths can be attributed to numerical effects in the linearized mixing length equations. For these models, the lower boundary of the convection zone is very close to the lower boundary of the atmosphere. Note that these differences are not visible in the spectra and that the differences are less than $1 \%$ (see temperature scale for the spherical $\left(T_{\text {eff }}, \log g\right)=(6500 \mathrm{~K}, 1.5)$ model at the top axis of the figure).

In the outermost layers, the temperature differences for models of equal $\log g$ and different $T_{\text {eff }}$ show a complex behaviour. In certain cases, models with larger extension show less surface cooling with respect to the plane-parallel counterpart than less extended models, for example the models with $\log g=1.5$ shown in Fig. 2. This is very likely due to the effect of the change in temperature structure on molecular absorption. An inspection of the grid models shows that the changes in partial pressures of molecules in the outermost layers, when going from planeparallel to spherical geometry, do not simply scale with effective temperature. This applies in particular to molecules containing carbon, as well as $\mathrm{TiO}$ and SiS. Since molecular absorption

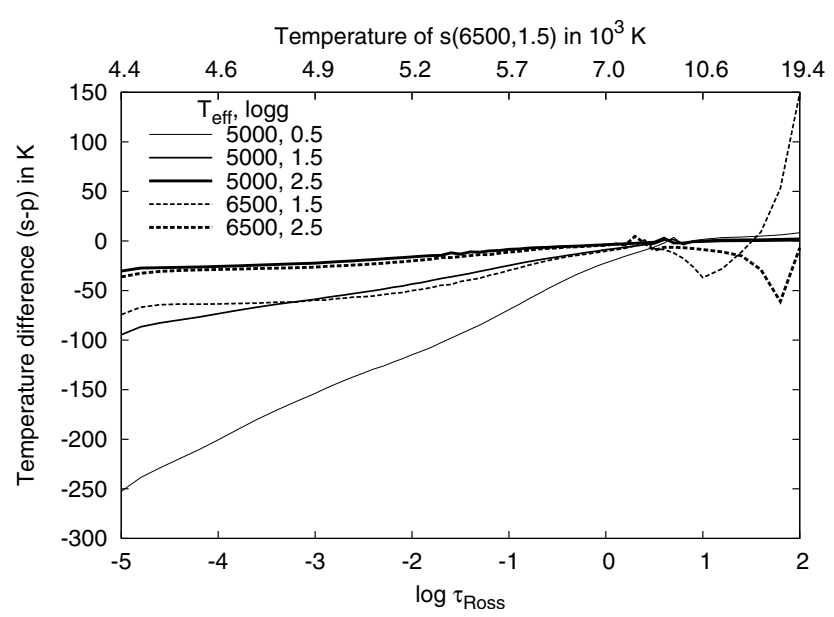

Fig. 2. Differences between spherical and plane-parallel model temperatures $\left(T_{s}-T_{p}\right)$ as a function of Rosseland optical depth of models with $\xi_{\mathrm{t}}=2 \mathrm{~km} \mathrm{~s}^{-1}$, solar abundances and $M=1 M_{\odot}$. Solid (dashed) lines show $T_{\text {eff }}=5000 \mathrm{~K}(6500 \mathrm{~K})$ models and line width increases with $\log g$. The scale on the top horizontal axis gives the temperature of the spherical $\left(T_{\mathrm{eff}}, \log g\right)=(6500 \mathrm{~K}, 1.5)$ model in units of $10^{3} \mathrm{~K}$.

can either cool or heat the outer layers (Gustafsson \& Olander 1979), the net effect might well be more cooling in a less extended model as compared to a more extended model.

\section{Models, spectra and equivalent widths}

We calculated spectra with two different versions of the spectrum synthesis code used in Uppsala (plane-parallel $-p-$ and spherical $-s$ ) for representative model parameters. We chose solar metallicity for all models and masses of 1,2 and $5 M_{\odot}$ for $s$ models. The microturbulence parameter $\xi_{\mathrm{t}}$ was set to 2 and $5 \mathrm{~km} \mathrm{~s}^{-1}$. $T_{\text {eff }}$ was varied between 4000 and $6500 \mathrm{~K}$ in steps of $500 \mathrm{~K}$ for $1 M_{\odot}$ and $1000 \mathrm{~K}$ for 2 and $5 M_{\odot}$. The $\log g$ values ranged from 0.5 to 3.0 in steps of 0.5 for $1 M_{\odot}$ and were set to $1.0,2.0$ and 3.0 for 2 and $5 M_{\odot}$. For $1 M_{\odot}$, this corresponds to radii at $\tau_{\text {Ross }}=1$ of $\approx 5 R_{\odot}(\log g=3.0), \approx 15 R_{\odot}(\log g=2.0)$ and $\approx 90 R_{\odot}(\log g=0.5)$; for 2 and $5 M_{\odot}$ and $\log g=2.0$, the radii are $\approx 20$ and $40 R_{\odot}$, respectively. For $\log g=0.5$, only models with $T_{\text {eff }} \leq 5000 \mathrm{~K}$ were available. Normalized synthetic spectra were calculated for wavelengths between 5400 and $7200 \AA$ Asing atomic line data from VALD (Kupka et al. 1999) and MARCS model structures (Gustafsson et al. 2003) ${ }^{2}$ as input. For a discussion of the method for solving the radiative transfer equations in the atmospheric models and spectrum synthesis see Plez et al. (1992) and Nordlund (1984). We regard three different combinations of model atmosphere (atm) and spectrum synthesis (syn) geometries: $a t m \_s y n=s \_s$ (consistently spherical), $s \_p$ (inconsistent), $p_{\_} p$ (consistently plane-parallel).

As a next step, we selected all lines in this spectral region which were found to be not blended by more than $30 \%$ by neighboring lines. This line selection was done for the $(5000 \mathrm{~K}, 0.5)$, $\xi_{\mathrm{t}}=2$ and $5 \mathrm{~km} \mathrm{~s}^{-1}, 1 M_{\odot} s_{-} s$ models. Equivalent widths were then calculated for these lines for the $s_{-} s$ models with an equivalent width / abundance fit version of the spectrum synthesis code. Finally, abundance differences were obtained for $s_{-} p$ and $p_{-} p$ models by fitting the calculated equivalent widths to the $s_{-} s$ ones. Table 1 lists all species contained in the line list and the number of lines selected for each of them, as well as the

\footnotetext{
2 http://marcs.astro.uu.se
} 
Table 1. Number of lines selected for each species in the geometrically consistent $\left(s \_s\right)$ synthetic spectrum with $\left(T_{\text {eff }}, \log g, \xi_{\mathrm{t}}\right)=(5000 \mathrm{~K}, 0.5$, $2 \mathrm{~km} \mathrm{~s}^{-1}$ ) with equivalent widths between 5 and $250 \mathrm{~m} \AA$, as well as fraction of the element in the given ionization stage (in \%, averaged over $\left.-1.0 \leq \log \tau_{\text {Ross }} \leq-0.1\right)$.

\begin{tabular}{lrrlrlllr}
\hline \hline Species & $n$ & $\%$ & Species & $n$ & $\%$ & Species & $n$ & $\%$ \\
\hline Al I & 9 & 0.1 & Gd II & 14 & 99.8 & Sc II & 15 & 99.9 \\
C I & 21 & 100.0 & La II & 33 & 98.6 & Si I & 173 & 5.2 \\
Ca I & 28 & 0.01 & Mg I & 8 & 0.5 & Si II & 4 & 94.8 \\
Ce II & 33 & 98.3 & Mn I & 17 & 0.5 & Sm II & 104 & 98.3 \\
Co I & 76 & 1.8 & Mn II & 3 & 99.5 & Ti I & 115 & 0.1 \\
Cr I & 41 & 0.1 & Mo I & 3 & 0.3 & Ti II & 34 & 99.9 \\
Cr II & 22 & 99.9 & Nd II & 109 & 96.7 & V I & 57 & 0.1 \\
Dy II & 5 & 99.3 & Ni I & 124 & 2.6 & V II & 21 & 99.9 \\
Er II & 5 & 99.6 & O I & 4 & 100.0 & Y II & 10 & 99.8 \\
Eu II & 7 & 98.6 & Pr II & 73 & 94.8 & Zr I & 6 & 0.1 \\
Fe I & 562 & 1.0 & S I & 21 & 91.1 & Zr II & 7 & 99.9 \\
Fe II & 68 & 99.0 & Sc I & 8 & 0.03 & & & \\
\hline
\end{tabular}

fraction of the element in the given ionization stage, averaged over $-1.0 \leq \log \tau_{\text {Ross }} \leq-0.1$.

\section{Results}

We take the consistent $s \_s$ spectra as a reference and present a comparison to the spectra with the two other geometry combinations. Figure 3 illustrates the geometry effects on line profiles. One can see that mainly the line centers are affected, which can be ascribed to the way the model atmosphere structure changes (see Fig. 2). The following two subsections summarize the effects on line abundances for $1 M_{\odot}$ models. We restrict the investigation to lines with equivalent widths between 5 and $250 \mathrm{~m} \AA$.

\subsection{Effects of inconsistent spectrum synthesis}

Figure 4 shows the differences between $s \_p$ and $s \_s$ abundances versus $s \_s$ equivalent widths $(W)$ for $\mathrm{Fe} I$ lines (upper plot) and Fe II lines (lower plot). Models with $T_{\text {eff }}=5000 \mathrm{~K}$, low microturbulence and various gravities are shown. Apart from the expected increase towards lower gravities, one notices that the effect is on average about $0.01 \mathrm{dex}$ smaller for $\mathrm{Fe}$ II than for Fe I, for lines with $W \lesssim 50 \mathrm{~m} \AA$ and $\log g \leq 1$. The same difference between ionization stages is seen for all other elements with at least 10 lines of each of the two ionization stages $(\mathrm{Cr}$, $\mathrm{Ti}, \mathrm{V})$. The differences are slightly larger for higher $T_{\text {eff }} \mathrm{s}$. This is illustrated in Fig. 5, which shows the differences for Fe lines for $\xi_{\mathrm{t}}=2 \mathrm{~km} \mathrm{~s}^{-1}, \log g=1.0$ and several $T_{\text {eff }}$ values, averaged over bins of $25 \mathrm{~m} \AA$. The trends are weaker for $\xi_{\mathrm{t}}=5 \mathrm{~km} \mathrm{~s}^{-1}$ and different for different species. The differences are in general larger for the lower $\xi_{\mathrm{t}}$ value, where line saturation sets in at smaller $W$. Neutral species show the same differences as Fe I, except for C I, S I and Si I, for which the effect is on average smaller by 0.01 dex for the lowest $\log g$ values and weak lines. Singly ionized species show the same differences as Fe II, except for weak lines of rare earth elements for $\log g=0.5$. For Ce II, the effect is on average smaller by $0.01 \mathrm{dex}$, and for Gd II, Pr II, Nd II, La II, Sm II, the effect is on average larger by $0.01 \mathrm{dex}$.

\subsection{Effects of consistent plane-parallel spectrum synthesis}

Figure 6 shows the differences between $p \_p$ and $s \_s$ abundances versus $s \_s$ equivalent widths for Fe I and Fe II lines, for the models with $T_{\text {eff }}=5000 \mathrm{~K}$, low microturbulence and various gravities. In this case, neutral and ionized Fe lines show an opposite effect, which is also seen for all other elements. The dependence of abundance differences on $T_{\text {eff }}$ is more complex than in the previous case (Sect. 3.1), as shown Fig. 4. For Fe I lines, the differences are essentially zero at $T_{\text {eff }}=4000 \mathrm{~K}$, increase up to $T_{\text {eff }}=5000 \mathrm{~K}$, and decrease slightly towards higher $T_{\text {eff }}$. For Fe II lines, the differences decrease with increasing $T_{\text {eff }}$ over the whole range.

Again, most neutral species behave similarly to Fe I, with the following exceptions:

- C I, O I and S I (all predominantly neutral): the effect has opposite sign to that of Fe I (similar to Fe II);

- Si I: the effect is less than half that of Fe I;

- Ti I and V I: the effect is on average larger than for Fe I.

Most singly ionized species show the same differences as Fe II. Exceptions are again the rare earth elements, but also Sc and Ti:

- Ce II, La II, Nd II, Sc II: the effect is smaller than for Fe II and changes sign for large equivalent widths;

- Sm II, Ti II: the effect is on average 0.01 dex smaller than for Fe II for $\log g \leq 1.0$.

\subsection{Mass dependence}

For models with $\left(T_{\text {eff }}, \log g, \xi_{\mathrm{t}}\right)=\left(5000 \mathrm{~K}, 1.0,2 \mathrm{~km} \mathrm{~s}^{-1}\right)$ and a mass of $2 M_{\odot}$, the abundance differences are smaller by 0.005 (weak lines) to $0.01 \mathrm{dex}$ (strong lines) compared to $1 M_{\odot}$ models. For a model mass of $5 M_{\odot}$, they are smaller by 0.01 (weak lines and strong lines of majority species in the $p_{-} p$ case) to 0.03 (strong lines in the $s_{-} p$ case) to $0.05 \mathrm{dex}$ (strong lines of minority species in the $p_{-} p$ case). In general, higher masses result in smaller effects and the dependence on mass is proportional to abundance difference. As can be seen in Fig. 1, the extensions in $5 M_{\odot}, \log g=1.0$ models are approximately the same as in $1 M_{\odot}, \log g=1.5$ models. We therefore expect that an increase in mass from 1 to $5 M_{\odot}$ has the same effect as increasing $\log g$ by 0.5 . This is confirmed by the calculations.

\section{Discussion}

\subsection{Trends of effects with line data}

It seems that the temperature structure used as input for the spectrum synthesis calculation is more important than the geometry used in such a code. Since the temperature differences decrease with depth in the atmosphere, spectral lines formed at different depths will be affected differently. We calculated average formation depths $\left\langle\tau_{\text {Ross }}\right\rangle$ of the line centers for a few species which show different effects (Sect. 3) for the $s$ model with $\left(T_{\text {eff }}\right.$, $\left.\log g, \xi_{\mathrm{t}}\right)=\left(5000 \mathrm{~K}, 0.5,2 \mathrm{~km} \mathrm{~s}^{-1}\right)$. The distributions (relative frequency of lines forming at different $\left.\log \left\langle\tau_{\text {Ross }}\right\rangle\right)$ are rather similar, except for those of C I, S I, Si I and Ti I. They show a somewhat higher and narrower peak at $\log \left\langle\tau_{\text {Ross }}\right\rangle \approx-0.3$ than that of Fe I. This will introduce a small species to species difference in the geometry effects.

The largest part of the differential effect can however be attributed to the different distributions of excitation energies over lines of the same species (see Sect. 4.2.3 below). Figures 8 and 9 

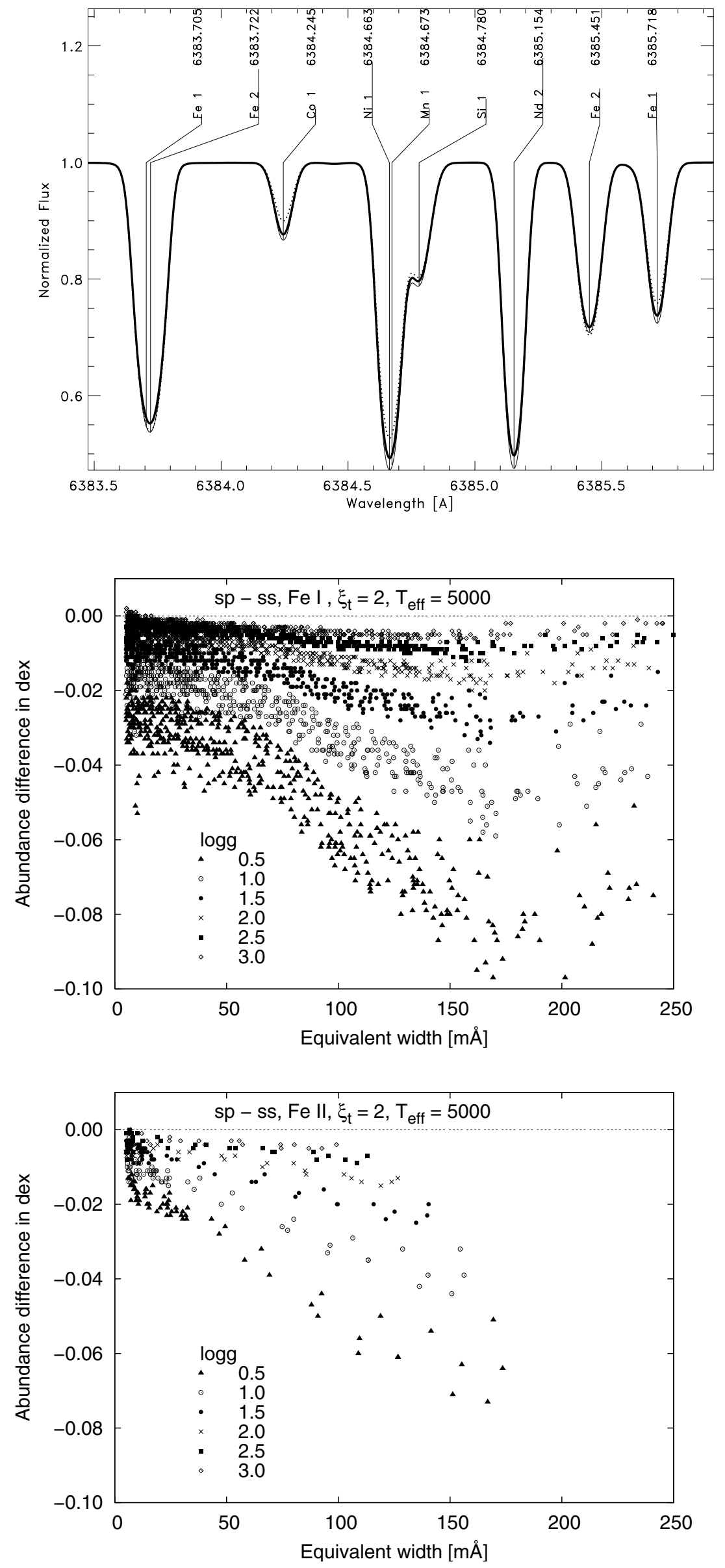

Fig. 3. Comparison of $s_{-} s$ (thick line), $s_{-} p$ (thin line) and $p_{-} p$ (dotted line) spectra for $\left(T_{\mathrm{eff}}\right.$, $\left.\log g, \xi_{\mathrm{t}}, M\right)=\left(5000 \mathrm{~K}, 0.5,2 \mathrm{~km} \mathrm{~s}^{-1}, 1 M_{\odot}\right)$.

Fig. 4. Abundance differences for inconsistent and consistently spherical equivalent widths of $\mathrm{Fe} \mathrm{I}$ lines (upper plot) and Fe II lines (lower plot) as a function of $s_{-} s$ equivalent widths for models with a mass of $1 M_{\odot}$ with $\xi_{\mathrm{t}}=2 \mathrm{~km} \mathrm{~s}^{-1}$, $T_{\text {eff }}=5000 \mathrm{~K}$, and different $\log g$ values as indicated by different symbols. 

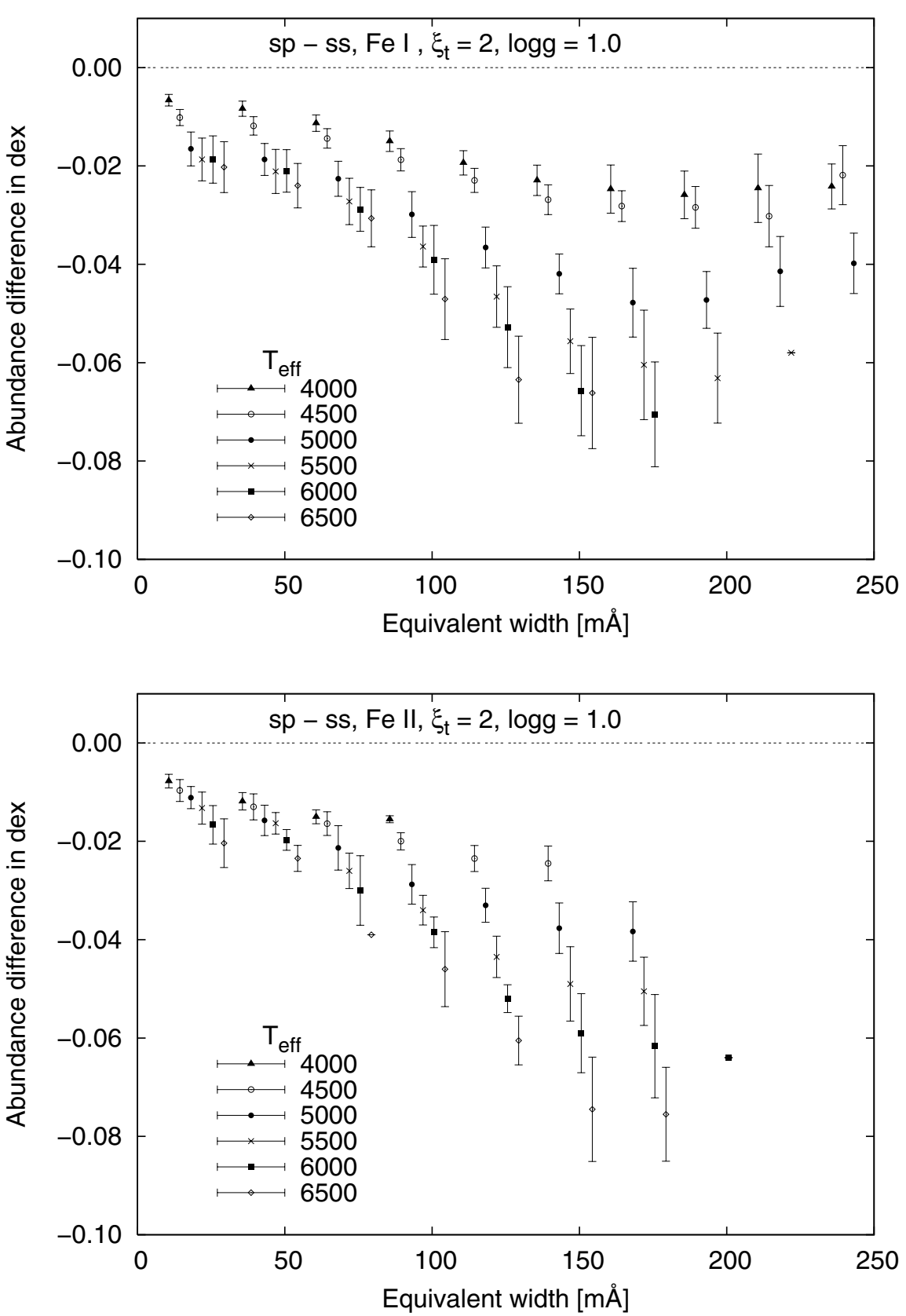

Fig. 5. Abundance differences for inconsistent and consistently spherical equivalent widths of $\mathrm{Fe} I$ lines (upper plot) and Fe II lines (lower plot) as a function of $s_{-} s$ equivalent widths for models with a mass of $1 M_{\odot}$ with $\xi_{\mathrm{t}}=2 \mathrm{~km} \mathrm{~s}^{-1}$, $\log g=1.0$, and different $T_{\text {eff }}$ values as indicated by different symbols. The differences have been averaged over bins of $25 \mathrm{~m} \AA$, with bin centers offset for clarity and error bars showing the standard deviations. show the correlations between excitation potential and abundance difference (for ionized species) as well as between the difference of ionization and excitation potentials and abundance difference (for neutral species).

\subsection{Analytical estimation of effects}

As giant stars with exactly plane-parallel atmospheres do not exist in nature, it will be difficult to verify these results with observations. A possible experiment would be to perform an abundance analysis of a sample of binary stars, where one component is a dwarf star close to the zero-age main sequence and the second components are giant stars of varying sizes. When both stars are analysed with a plane-parallel spectrum/atmosphere code, any difference in abundance could be attributed to sphericity effects. However, this requires that all other physical phenomena, like convection, deviation from LTE and scattering, are modelled correctly. Here, in order to conduct a "sanity check" on the results, we try to reproduce them using an analytical solution to the problem. This will also help to get a better understanding for the cause of the effects. Such solutions require invoking a few more approximations than used in the numerical model. We follow Chandrasekhar (1934), who derived a solution to the equation of radiative transfer (in polar coordinates), including the term containing the dependence of intensity $I$ on the angle of inclination to vertical $\theta$

$\cos \theta \frac{\partial I}{\partial r}-\frac{\sin \theta}{r} \frac{\partial I}{\partial \theta}=\kappa \rho(S-I)$,

where $r$ is the distance from the stellar center (i.e., the radius), $\kappa$ is the opacity, $\rho$ the density and $S$ the source function. In local thermodynamic equilibrium (LTE), $S$ is given by the Planck function $B$. Further, if the mean intensity $J$, the flux $\pi F$, and the radiation pressure integral $K$ are defined as the zeroth, first and second moment of the intensity, respectively, the first Eddington approximation $3 K=J$ is used, along with the 

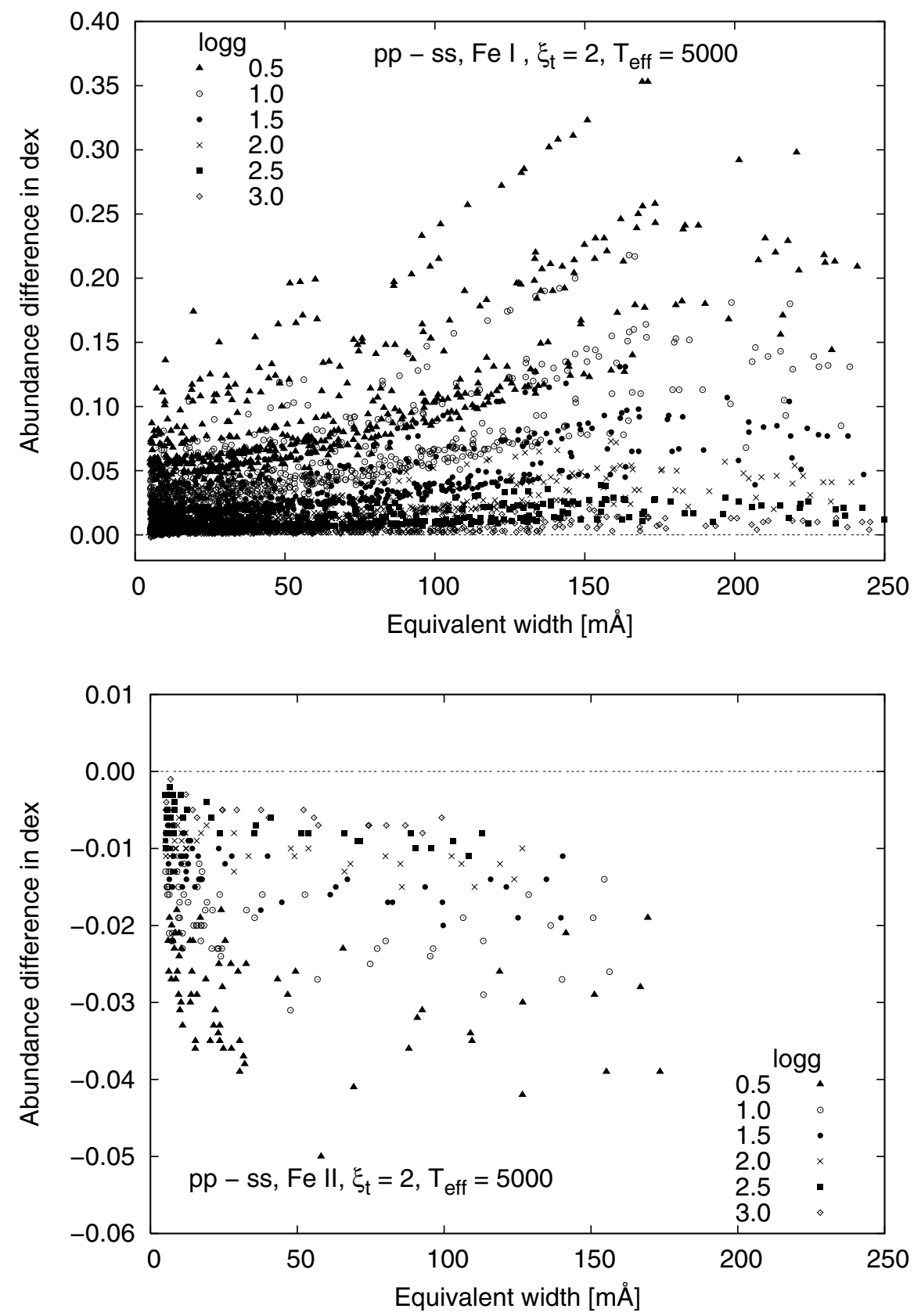

Fig. 6. Differences between consistently planeparallel and spherical abundances versus $s_{-} s$ equivalent widths of $\mathrm{Fe}$ I lines (upper plot) and Fe II lines (lower plot) for models with a mass of $1 M_{\odot}$ with $\xi_{\mathrm{t}}=2 \mathrm{~km} \mathrm{~s}^{-1}, T_{\text {eff }}=5000 \mathrm{~K}$, and different $\log g$ values as indicated by different symbols. condition that at the outer boundary of the star $F=2 J$ (second Eddington approximation). Radiative equilibrium is expressed by $F r^{2}=$ constant. The dependence of $B$ on optical depth $\tau$ is then

$B=\frac{1}{2} F_{R}\left(1+\frac{3}{2} \int_{0}^{\tau} \frac{R^{2}}{r^{2}} \mathrm{~d} \tau\right)$,

where $\pi F_{R}$ is the flux at $r=R$, which occurs at $\tau=0$. Note that this definition of $R$ is different from the radii given in Sect. 2 . If $\tau_{\text {Ross }}=10^{-5}$ is taken as an approximation for $\tau=0, R$ is the radius at $\tau_{\text {Ross }}=1$ enlarged by the corresponding extension (Fig. 1). We adopt this approach in the following.

The same approximations as above are subsequently used when considering the formation of absorption lines. Now, all radiation quantities are indexed with frequency $v$ to indicate that they are defined in the range $v$ to $v+\mathrm{d} v$ in continuum regions, and additional primes indicate quantities inside spectral lines.

\subsubsection{The s s case}

Chandrasekhar (1934) assumed a power law variation of $\tau_{v}$ with $r$

$$
\tau_{v}=c r^{m}
$$

where $c$ and $m$ are constants and $m<0$. Furthermore, $R$ was assumed to be sufficiently large so that

$\frac{1}{R^{2}} \ll \frac{3}{2} \int_{0}^{\tau_{v}} \frac{\mathrm{d} \tau_{v}}{r^{2}}$

Assuming small $|m|$, he derived a surprisingly simple expression for the dependence of normalized line flux on line strength, the latter being measured by the ratio of the coefficients of line to continuous absorption $\eta$,

$\frac{F_{v}^{\prime}}{F_{v}}=\frac{1}{1+\eta}$ 

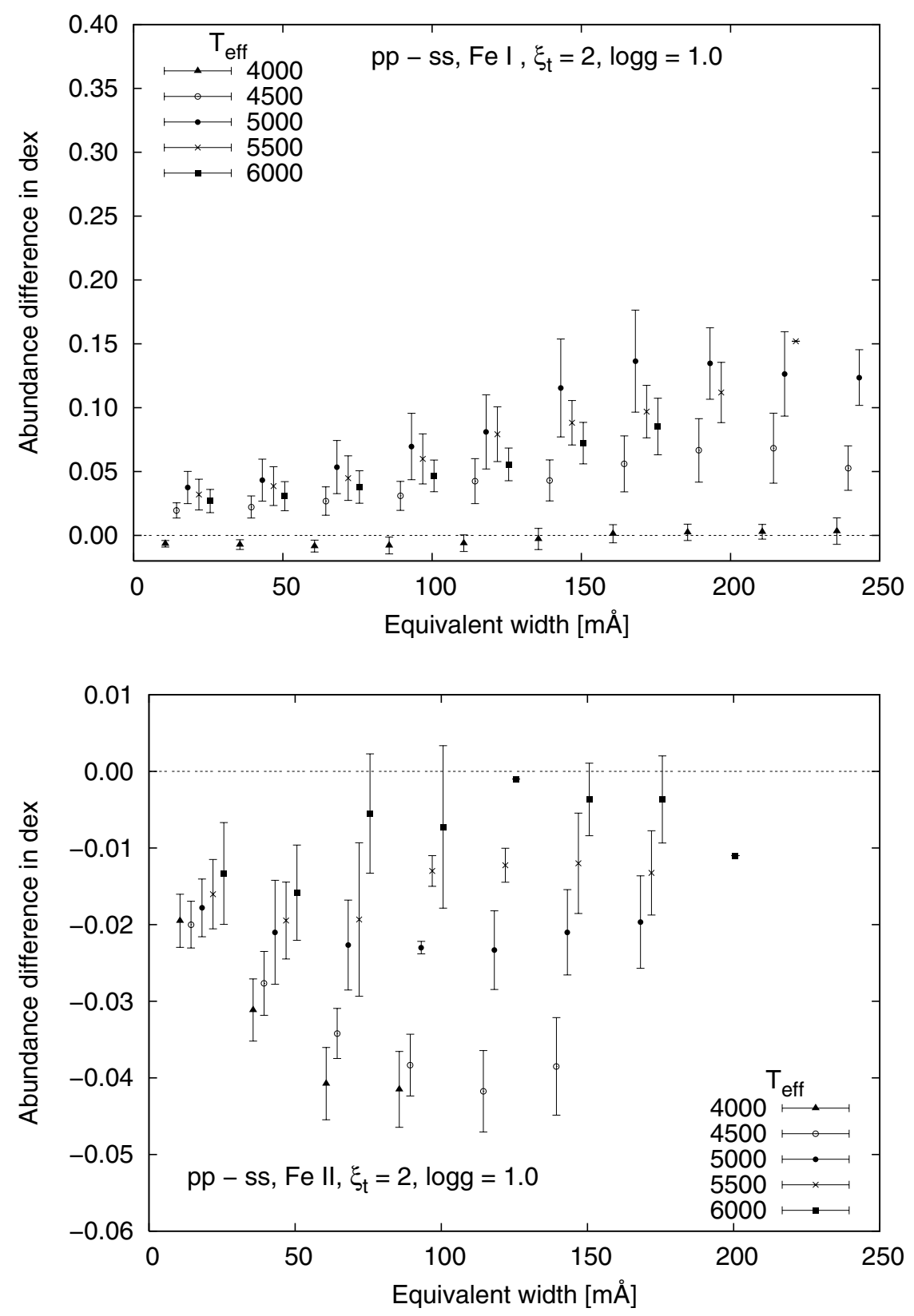

Fig. 7. Differences between consistently planeparallel and spherical abundances versus $s_{-} s$ equivalent widths of Fe I lines (upper plot) and Fe II lines (lower plot) for models with a mass of $1 M_{\odot}$ with $\xi_{\mathrm{t}}=2 \mathrm{~km} \mathrm{~s}^{-1}, \log g=1.0$, and different $T_{\text {eff }}$ values as indicated by different symbols. The differences have been averaged over bins of $25 \mathrm{~m} \AA$, with bin centers offset for clarity and error bars showing the standard deviations.
Equation (5) presumably describes the $s \_s$ case and can be directly compared to a similar expression derived by Eddington (1929) for the plane-parallel case

$$
\frac{F_{v}^{\prime}}{F_{v}}=\frac{1+\frac{2}{3} q}{1+\eta+\frac{2}{3} q} .
$$

Here, $q^{2}=3(1+\eta)(1+\epsilon \eta)$ includes a parameter $\epsilon$ measuring the importance of collisions (i.e. $\epsilon=1$ in LTE). Comparing the two expressions, it becomes clear that Eq. (5) by far overestimates the effect of sphericity, since the normalized line flux decreases much more rapidly with line strength than in Eq. (6), and it approaches zero for large $\eta$, whereas in Eq. (6), the limiting value is $1 /(1+\sqrt{3} / 2)=0.54$. Thus, Eq. (5) cannot be used to estimate the effects for the parameter range studied here. An inspection of the calculated models shows that neither of the two assumptions used by Chandrasekhar (1934) are fulfilled. We determine values of $m$ and $c$ by fitting the function given by Eq. (3) to the calculated values of $\tau_{5000} \AA$ and $r$ in the range
$-5 \leq \log \tau_{5000 \AA} \leq 0$. The ratio of $\frac{1}{R^{2}}$ and the right hand side of Eq. (4) is then between 0.5 to 0.7 when using $\tau_{5000 \AA}=1$ as upper integration limit and correspondingly larger for smaller upper integration limits. Furthermore, the values of $|m|$ are rather large (between 100 and 2000).

\subsubsection{The s_p case}

For the $s \_p$ case we start from a slightly different expression for the normalized line flux, derived for the case where the continuum source function varies linearly with optical depth $\left(S_{v}=B_{v}=a+b \tau_{v}\right)$ :

$\frac{F_{v}^{\prime}}{F_{v}}=\frac{b+a q}{b+a \sqrt{3}} \cdot \frac{1+\frac{2}{3} \sqrt{3}}{1+\eta+\frac{2}{3} q}$

(see Eddington 1929, his Eq. (22)).

The effect of using a spherical model atmosphere compared to a plane-parallel one can then be estimated by comparing 

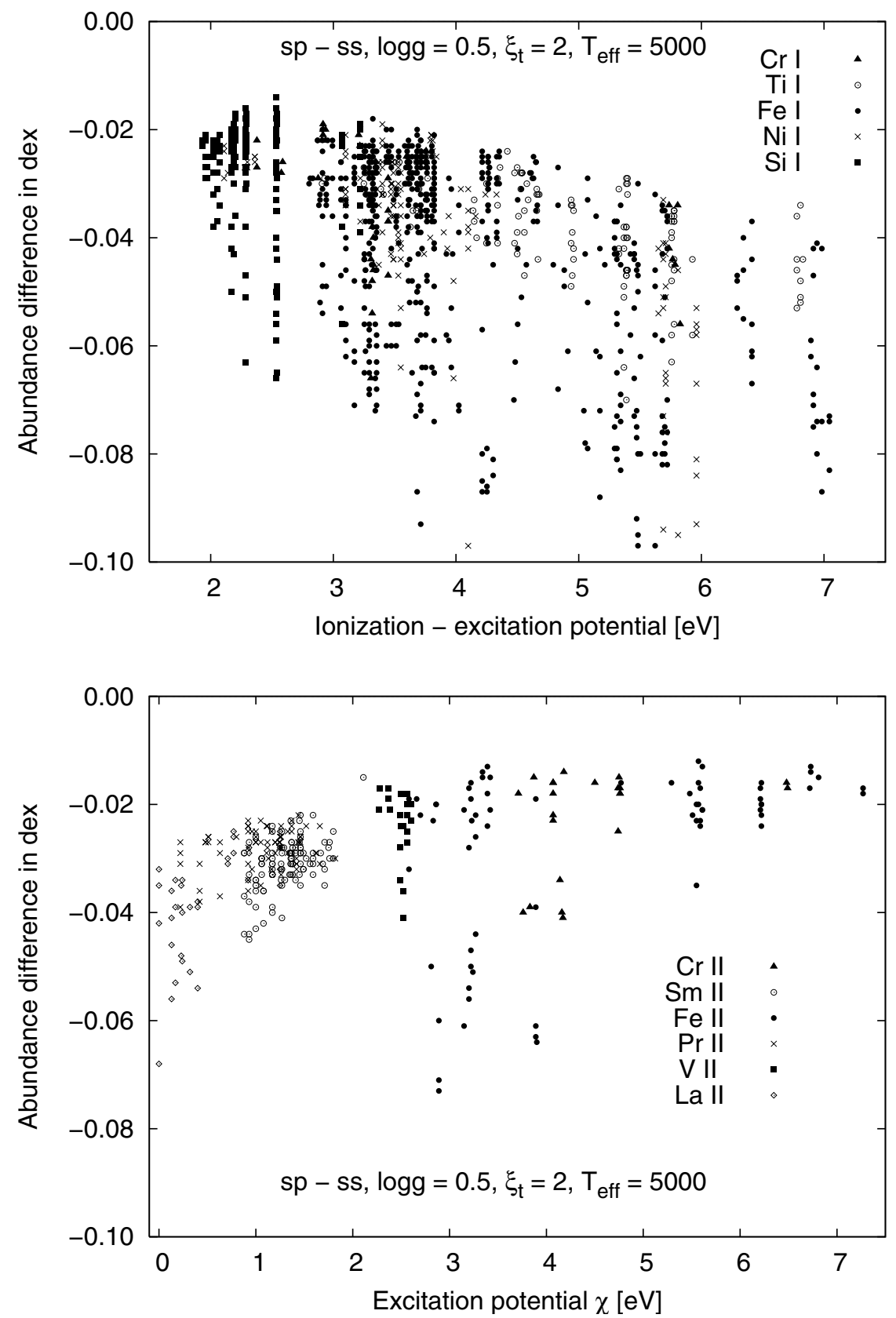

Fig. 8. Abundance differences $s_{-} p-s_{-} s$ versus difference of ionization and excitation potentials (neutral species, upper plot) and versus excitation potential (singly ionized species, lower plot). corresponding numerical values of the ratio $b / a$. In LTE, $S_{v}=$ $B_{v}=C_{v} /\left(\mathrm{e}^{x}-1\right)$, where $C_{v}$ is constant for a given frequency and $x=h v / k T, T$ being the temperature and $h$ and $k$ the Planck and Boltzmann constants, respectively. The derivative of the logarithm of the Source function with respect to $\tau_{v}$ is then

$\frac{1}{S_{v}} \frac{\mathrm{d} S_{v}}{\mathrm{~d} \tau_{v}}=\frac{x \mathrm{e}^{x}}{\mathrm{e}^{x}-1} \frac{1}{T} \frac{\mathrm{d} T}{\mathrm{~d} \tau_{v}}$.

For a grey atmosphere, where the continuous absorption is independent of $v$, the temperature structure is given by $T^{4} \propto\left(1+\frac{3}{2} \tau\right)$ in the plane-parallel case. Inserting in Eq. (8) together with the linear source function law, we derive $b / a=\frac{3}{8} x$ at $\tau=0$. This expression only holds for $x \gtrsim 3$, for which $\mathrm{e}^{x} /\left(\mathrm{e}^{x}-1\right)$ can be approximated by 1 . Note that the lowest value of $x$ occurring in the atmospheric models studied here is about 3 (for $7200 \AA$ and the hottest models at $\tau_{\text {Ross }}=1$ ). In the spherical case, using Eq. (2), the grey temperature structure can be written as $T^{4} \propto\left(1+\frac{3}{2} \int_{0}^{\tau} \frac{R^{2}}{r^{2}} \mathrm{~d} \tau\right)$. We use the same law for $\tau(r)$ as above (Eq. (3)) to evaluate the integral in the temperature structure equation and further the derivative in Eq. (8). For large values of $|m|$, this leads to $b / a=\frac{3}{8} x c^{2 / m} R^{2}$. In order to estimate numerical values of the discriminating factor $c^{2 / m} R^{2}$, we resort to determining them from the atmospheric models: for $\log g$ values of $0.5,1.5$ and 2.5 , typical values of the factor are 1.2, 1.1, and 1.02 , respectively. The resulting variation in normalized line flux is shown in Fig. 10 as a function of line strength $\eta$, for a value of $x=4$ (corresponding to e.g. a wavelength of $6000 \AA$ at a temperature of $6000 \mathrm{~K}$ ). Also shown is the relation for the $p \_p$ case (Eq. (6), which derives from Eq. (7) when $x=4$ ). Comparing this figure to Fig. 3 shows that the analytic estimation of the sphericity effect agrees fairly well with the modelling 

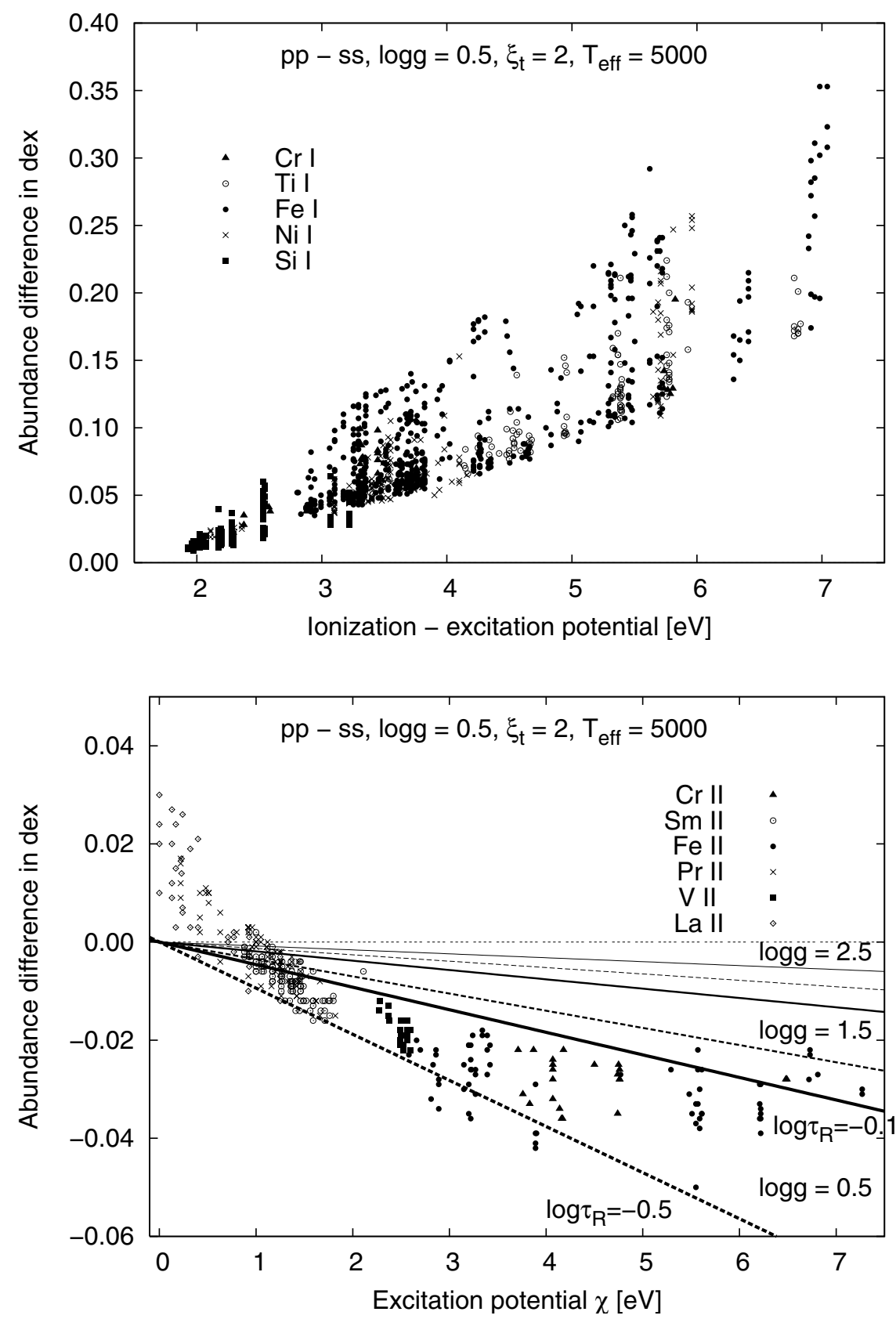

Fig. 9. Abundance differences $p \_p-s_{-} s$ versus difference of ionization and excitation potentials (neutral species, upper plot) and versus excitation potential (singly ionized species, lower plot). The lines in the lower plot indicate the variation of abundance difference as estimated from the difference in model temperature only (see text, Sect. 4.2.3) at $\log \tau_{\text {Ross }}=-0.5$ (dashed) and $\log \tau_{\text {Ross }}=-0.1$ (solid), for different $\log g$ values as indicated by different line width. outcome in this case, at least for lines of species in the minority ionization stage.

The results of this discussion suggest another observational test. When central depths of saturated lines are measured in giant stars of different radii, they are relatively unaffected by star-tostar abundance differences. Those lines having cores that form close to LTE should then be deeper for the more extended stars than for the less extended stars by the amount predicted.

\subsubsection{Dependence on excitation potential}

The variation of the abundance differences with excitation potential $(\chi)$ can under certain circumstances be directly estimated from model temperature differences. We use the assumption that for a weak line of a given species, changes in logarithmic abundance are equivalent to changes in $5040 / T \cdot \chi$ for a line from the majority species (see e.g. Gray 1992, Chap. 14). The temperature difference in the region of $\log \tau_{\text {Ross }}$ between -0.1 and -0.5 , where most of the lines form, is about 30,15 and $5 \mathrm{~K}$ for models with $\log g=0.5,1.5$ and 2.5 , respectively. The corresponding variations of abundance difference with $\chi$ are shown in Fig. 9 (lower plot) as lines and are comparable to the outcome of the detailed models. For lines of species in the minority ionization stage (all neutral elements except C I, O I and S I, see Table 1), the differences will also depend on the ionization potential.

\subsection{Implications for abundance analysis}

The systematically different effects on lines of different ionization stages of the same element imply an effect on ionization equilibrium, which is sometimes used to infer gravities of program stars. Just as an example, we take the star YZ Sgr from Luck \& Andrievsky (2004). At phase 0.249 it has a temperature of $5500 \mathrm{~K}, \log g=1.4$ and $\xi_{\mathrm{t}}=3.7 \mathrm{~km} \mathrm{~s}^{-1}$. The average abundance difference $\left(p \_p-s \_s\right)$ for Fe I lines used by Luck \& Andrievsky (2004), which are also in our line list, amounts 


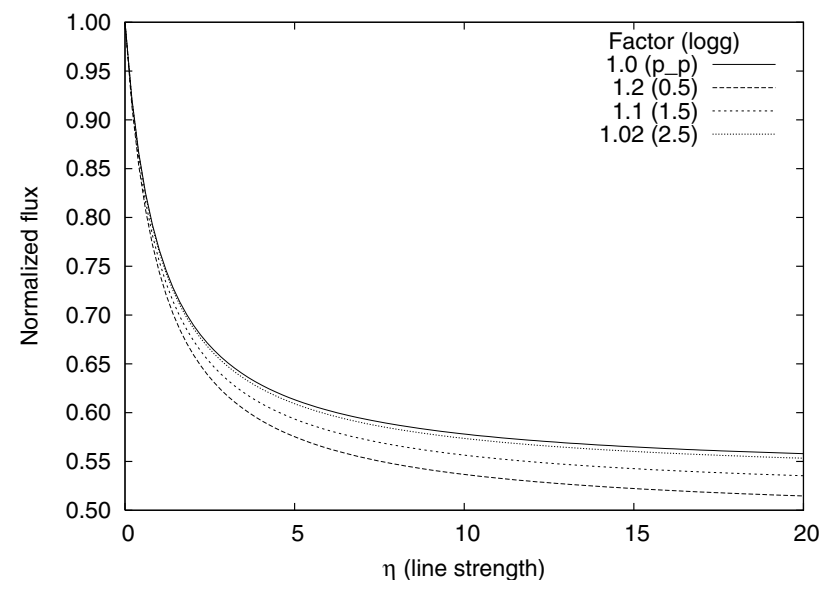

Fig. 10. Normalized flux as a function of line strength showing the $s \_p$ effect as estimated according to Eq. (7), where $b / a=\frac{3}{8} x c^{2 / m} R^{2}$, for $x=$ 4 and three different factors $c^{2 / m} R^{2}$ determined from the atmospheric models for different $\log g$ values.

to $+0.03 \pm 0.01 \mathrm{dex}$ (for the $5500 \mathrm{~K} / 1.5$ model with $\xi_{\mathrm{t}}=2$ or $5 \mathrm{~km} \mathrm{~s}^{-1}$ ). For Fe II, the difference is $-0.010 \pm 0.003$ dex. The same difference is obtained by decreasing $\log g$ in the $s_{-} s$ models by about 0.1 dex or increasing the temperature by about $40 \mathrm{~K}$. These values are equal to the formal uncertainty given for this star. In the Takeda et al. (2005) sample, stars at $5000 \mathrm{~K}$ have $\log g \geq 2.5$. If we take as an example the star HD 192944 $\left(\xi_{\mathrm{t}}=1.4 \mathrm{~km} \mathrm{~s}^{-1}\right)$, the abundance differences $\left(p \_p-s_{-} s\right)$ are only $+0.009 \pm 0.006$ dex and $-0.008 \pm 0.004$ dex for Fe I and Fe II, respectively. They can safely be ignored until abundance analyses reach significantly higher internal accuracies compared to today. Abundance differences for individual lines and/or stellar parameters can be obtained on request from the authors.

\section{Conclusions}

1. We recommend the use of spherical model atmospheres in abundance analyses for $\log g \lesssim 2$ and $4000 \mathrm{~K} \leq T_{\text {eff }} \leq$ $6500 \mathrm{~K}$. Thus one can avoid both systematic errors on abundances and differential effects, which can lead to additional uncertainties in stellar parameters. Alternatively, restricting the analysis to weak lines $(W \lesssim 100 \mathrm{~m} \AA$ ) and/or majority species will minimize the uncertainties on abundances caused by assuming a plane-parallel geometry.

2. Geometry has a smaller effect on line formation than on model atmosphere structure. It is more important to use a spherical model atmosphere than to be consistent when calculating the spectral lines, i.e. an $s_{-} p$ calculation is better than $p_{-} p$ if tools for $s_{-} s$ calculations are not available.
Note, however, that this holds only for the range of parameters studied here. For a model of a cool giant with $\left(T_{\text {eff }}\right.$, $\log g)=(2800 \mathrm{~K},-0.6)$ for example, Aringer (2005) showed that an inconsistent treatment of geometry $\left(s_{-} p\right)$ results in larger differences in spectral line depth than for a fully planeparallel calculation.

3. For both cases ( $s_{-} p$ and $p_{-} p$ ), the results presented in Sect. 3 are intended to provide a guide to the estimation of systematic errors in abundance analyses due to geometry effects. For $s_{-} p$ calculations, the largest differences encountered with respect to the $s_{-} s$ case are -0.1 dex. In the $p_{-} p$ case, differences are up to +0.35 dex for minority species and at most -0.04 dex for majority species. The abundance studies of halo stars mentioned in Sect. 1 contain stars in the $\log g$ range where sphericity effects are important. Although the abundance difference values given in Sect. 3 are based on solar abundance models, effects are expected to be similar in low-metallicity models, since the atmospheric extensions are similar.

Acknowledgements. We thank all people who have contributed to the development of the current Uppsala version of the MARCS code throughout many years. We thank B. Edvardsson for calculating the grid of MARCS models used for the present work. B. Gustafsson, R.E. Luck, and the referee, C.I. Short, are thanked for several useful comments.

\section{References}

Andersen, J. 1999, Transactions of the IAU, Vol. XXIIIB (Kluwer Academic Publishers), 141

Andrievsky, S. M., Luck, R. E., Martin, P., \& Lépine, J. R. D. 2004, A\&A, 413, 159

Aringer, B. 2005, in Proc. ESO Workshop: High-resolution IR spectroscopy in Astronomy, ed. H. Käufl, R. Siebenmorgen, \& A. Moorwood

Chandrasekhar, S. 1934, MNRAS, 94, 444

Eddington, A. S. 1929, MNRAS, 89, 620

Gray, D. F. 1992, The observation and analysis of stellar photospheres (Cambridge University Press)

Gustafsson, B., Edvardsson, B., Eriksson, K., et al. 2003, in Stellar Atmosphere Modeling, ASP Conf. Ser., 288, 331

Gustafsson, B., \& Olander, N. 1979, Phys. Scr., 20, 570

Hauschildt, P. H., Allard, F., Ferguson, J., Baron, E., \& Alexander, D. R. 1999, ApJ, 525, 871

Kupka, F., Piskunov, N., Ryabchikova, T. A., Stempels, H. C., \& Weiss, W. W. 1999, A\&AS, 138, 119

Luck, R. E., \& Andrievsky, S. M. 2004, AJ, 128, 343

McWilliam, A., Preston, G. W., Sneden, C., \& Searle, L. 1995, AJ, 109, 2757

Nordlund, A. 1984, in Methods in Radiative Transfer, ed. W. Kalkofen (Cambridge University Press), 211

Plez, B. 1990, Mem. Soc. Astron. It., 61, 765

Plez, B., Brett, J. M., \& Nordlund, A. 1992, A\&A, 256, 551

Ryan, S. G., Norris, J. E., \& Beers, T. C. 1996, ApJ, 471, 254

Simmerer, J., Sneden, C., Cowan, J. J., et al. 2004, ApJ, 617, 1091

Takeda, Y., Sato, B., Kambe, E., et al. 2005, PASJ, 57, 109

Travaglio, C., Galli, D., \& Burkert, A. 2001, ApJ, 547, 217 